\title{
Comment
}

\section{Perfect high throughput screening assay: a crucial technique for drug discovery}

\author{
Guan-hua DU ${ }^{1}$ \\ Institute of Materia Medica, Peking Union Medical College \& Chinese Academy of Medical Sciences, Beijing 100050, China \\ ${ }^{1}$ Correspondence to Prof Guan-hua DU. Phn 86-10-6316-5184. Fax 86-10-6301-7757. E-mail dugh@imm.ac.cn
}

Received 2005-08-14

Accepted 2005-08-24

doi: $10.1111 / \mathrm{j} .1745-7254.2005 .00215 . \mathrm{x}$

Since being developed approximately 20 years ago, high throughput screening (HTS) has become one of the key techniques used in drug discovery ${ }^{[1]}$. However, three main problems are recognized with the use of HTS; namely, with the compound library, drug targets, and assay methods. Until now, the compound library has evolved based on the techniques of combinatorial chemistry and modern phytochemistry. Several functional proteins have emerged following the advance of genomics and proteomics. However, although many functional proteins have been discovered recently, they are not, as sometimes claimed, real drug targets; at best, they might be potential drug targets. The ideal targets selected for drug screening should qualify as drug targets $^{[2]}$. The selection of targets for drug screening is a crucial procedure in drug screening.

However, the main problem for drug discovery is in finding drugs or candidates based on the targets and compounds $s^{[3]}$. There are many different techniques used in drug discovery, such as virtual screening, and computer aid molecular design. However, all of the active compounds should be evaluated using biological methods. Therefore, establishing a novel biological assay is very important for drug screening in HTS. The assays used in HTS require high sensitivity and high stability, and must be performed automatically. The homogeneous assay is a simple and useful method for drug screening. It is suitable for automatic performance and can enhance the efficiency of screening, especially in HTS. Therefore, the homogenous system has become the favored technique in HTS.

The basic goal of small-molecule screening is to identify chemically "interesting" starting points for elaboration towards a drug ${ }^{[4]}$. The establishment of new screening assays will promote the discovery of lead compounds or candidates ${ }^{[5]}$. The HTS has been used in China since 1998 and, since then, experts have attempted to resolve some key problems with
HTS, such as with target validation, compound collection, and assay establishment ${ }^{[6]}$. In the current issue of Acta Pharmacologica Sinica, Dr Ming-wei WANG et al. introduced a homogeneous high throughput drug screening assay based on scintillation proximity assay technology for the identification of ligands of subunit acetylcholine recep-

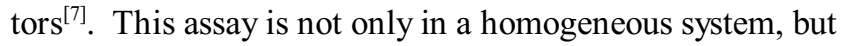
has a new target, a subunit of the acetylcholine receptor. The assay of homogeneous binding for $\alpha 4 \beta 2$ nicotinic acetylcholine receptor is a new assay for HTS to screen for ligands of $\alpha 4 \beta 2$ nicotinic acetylcholine receptors. This assay, with state-of-the-art design and interesting targets can be performed easily and automatically. The lead compound is valuable for new drug development and might also be used for validating the functions of the $\alpha 4 \beta 2$ nicotinic acetylcholine receptor. Screening with this assay might help us find new drugs with new mechanisms.

\section{References}

1 Bleicher KH, Bohm HJ, Muller K, Alanine AI. Hit and lead generation: beyond high-throughput screening. Nat Rev Drug Discov 2003; 2: 369-78.

2 Du GH, Liu AL. Application of bioinformatics in the high throughput screening. In: Zhen YS, editor. Anticancer drug research and development. Beijing: Chemical Industrial Press; 2004. p199216.

3 Archer JR. History, evolution, and trends in compound management for high throughput screening. Assay Drug Dev Technol 2004; 2: 675-81.

4 Walters WP, Namchuk M. Designing screens: how to make your hits a hit. Nat Rev Drug Discov 2003; 2: 259-66.

5 Comess KM, Schurdak ME. Affinity-based screening techniques for enhancing lead discovery. Curr Opin Drug Discov Devel 2004; 7: 411-6.

6 Du GH. High throughput pharmaceutical screening in China. Acta Pharm Sin 2000; 35 (Suppl): 14-7.

7 Hui X, Gao J, Xie X, Suto N, Ogiku T, Wang MW. A robust homogeneous binding assay for $\alpha 4 \beta 2$ nicotinic acetylcholine receptor. Acta Pharmacol Sin 2005: 26: 1175-80. 